\title{
Diagnóstico tardio de micose fungoide: um relato de caso
}

Late diagnosis of mycosis fungoides: a case report

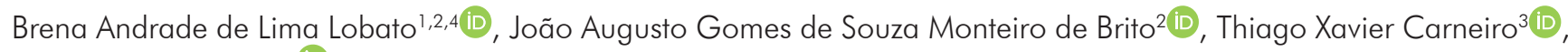 \\ Marília Brasil Xavier ${ }^{2,4}$ (D) \\ ' Universidade Federal do Pará, Núcleo de Medicina Tropical, Programa de Pós-Graduação em Doenças Tropicais, Belém, Pará, Brasil \\ 2 Universidade do Estado do Pará, Centro de Ciências Biológicas e da Saúde, Laboratório de Pesquisas em Dermatoses de Interesse Sanitário, Belém, \\ Pará, Brasil \\ ${ }^{3}$ Hospital Ophir Loyola, Divisão de Hematologia-Oncologia e Transplante de Células-Tronco, Belém, Pará, Brasil \\ ${ }^{4}$ Universidade Federal do Pará, Núcleo de Medicina Tropical, Laboratório de Pesquisas em Dermatologia Tropical e Doenças Endêmicas, Belém, Pará, \\ Brasil
}

\section{RESUMO}

INTRODUÇÃO: Micose fungoide (MF) é um tipo de linfoma cutâneo de células T com incidência aproximada de 0,41/100.000 indivíduos anualmente. O presente relato ressalta os impactos do atraso diagnóstico na evolução da doença. RELATO DO CASO: Paciente do sexo feminino buscou assistência médica no serviço de dermatologia da Universidade do Estado do Pará (UEPA), em novembro de 2017, apresentando três placas eritemato-hipercrômicas infiltradas, duas no abdômen e uma descamativa no membro inferior esquerdo, e linfonodo palpável na região inguinal esquerda com crescimento progressivo que iniciou em 2011 . A paciente relatou que havia sido submetida a vários exames e tratamentos, assistida por seis profissionais médicos de diferentes especialidades e realizado quatro exames histopatológicos. O caso foi investigado no serviço de dermatologia, tendo sido realizado estudo imunohistoquímico e concluído o diagnóstico de MF. A paciente iniciou tratamento com metotrexato $15 \mathrm{mg}$ e ácido fólico $5 \mathrm{mg}$, em dose única semanal, e fototerapia com PUVA, tendo apresentado melhora significativa, porém, com extensa área de atrofia e infecções secundárias. CONCLUSÃO: O presente caso é um problema de saúde pública que reforça a necessidade de capacitação adequada de médicos para identificação e tratamento precoce de MF.

Palavras-chave: Micose Fungoide; Perfil de Impacto da Doença; Diagnóstico Tardio; Acesso aos Serviços de Saúde.

\begin{abstract}
INTRODUCTION: Mycosis fungoides (MF) is a cutaneous T-cell lymphoma with an incidence of approximately 0.41/100,000 individuals annually. This report highlights the impacts of delayed diagnosis on the evolution of the disease. CASE REPORT: A female patient sought medical assistance at the dermatology service of the Universidade do Estado do Pará (UEPA), Brazil, on November 2017, due to three infiltrated erythematous-hyperchromic plaques, two on the abdomen and one scaly on the left lower limb, and a palpable lymph node in the left inguinal region with progressive growth which started in 2011. The patient reported that she had undergone several tests and treatments and was seen by six medical doctors of different specialties and underwent four histopathological exams. The patient's case was investigated in the dermatology service of UEPA; an immunohistochemical study was performed, concluding the diagnosis of MF. She was treated with methotrexate $15 \mathrm{mg}$ and folic acid $5 \mathrm{mg}$ in a single weekly dose and phototherapy with PUVA, showing significant improvement after treatment, but with an extensive area of atrophy and secondary infections. CONCLUSION: The present case is a public health issue that reinforces the need for adequate medical training for early identification and treatment of MF.
\end{abstract}

Keywords: Mycosis Fungoides; Health Impact Assessment; Delayed Diagnosis; Health Services Accessibility. 


\section{INTRODUÇÃO}

A micose fungoide (MF), juntamente com a síndrome de Sezary, é classificada como o tipo mais comum de linfoma cutâneo de células T (LCCT), por representar de 44 a $54 \%$ dos casos. A doença acomete principalmente adultos, com idade média entre 55 e 60 anos, mas também pode ser encontrada em crianças e adolescentes. Ocorre na proporção de 1,6-2,0 homens para 1,0 mulher ${ }^{1,2}$.

A causa da MF ainda não está totalmente esclarecida, mas evidências sugerem que ocorra devido à estimulação antigênica crônica que pode gerar proliferação e acúmulo de células T na pele. Esse estímulo sofre grande influência do microambiente cutâneo, incluindo células dendríticas e células $T$ reativas citotóxicas ou regulatórias ${ }^{3}$. Os linfócitos T envolvidos na etiopatogenia da MF são as células $T$ CD4+ que apresentam moléculas de adesão, como CCR4 e CLA. Antígenos como CD7, ou eventualmente CD5 ou CD2, estão reduzidos ou ausentes nas células patogênicas ${ }^{4}$.

Clinicamente, os pacientes com MF apresentam, no início, lesões discretas que se assemelham ao eczema ou eritema difuso, podendo evoluir para o estágio tumoral, no qual decorre maior infiltração das lesões e, normalmente, ulceração. Essas lesões muitas vezes ocorrem em áreas que não são normalmente expostas ao sol, como o tronco, e podem se manifestar com hipocromia ou hipercromia ${ }^{5}$ Vale ressaltar que o diagnóstico da presente patologia é realizado considerando tanto critérios clínicos quanto histopatológicos ${ }^{6}$.

O tratamento é feito de acordo com o estadiamento da doença, de modo que, em estágio inicial (IA até IIA), são utilizadas terapias direcionadas à pele (TDP), como corticosteroides tópicos, radioterapia e fototerapia, e, em estágio avançado (IIB até IVB), é aplicada a terapia sistêmica com retinoides, interferons ou imunobiológicos, aliada às $\operatorname{TDP}^{7,8}$.

\section{DESCRIÇÃO DO CASO}

Paciente do sexo feminino, 56 anos de idade, do lar, natural e procedente de Belém, estado do Pará, procurou o serviço de dermatologia da Universidade do Estado do Pará (UEPA), em novembro de 2017, devido ao aparecimento de mancha escura de crescimento progressivo em membro inferior esquerdo (MIE), que se iniciara em maio de 2011. A paciente também relatou episódios de "inchaço" e dor local, além do aparecimento de duas novas manchas no abdômen e "íngua" na região inguinal esquerda. Informou ter sido submetida a vários exames e tratamentos, porém sem melhora e sem conclusão diagnóstica. Nesse contexto, a paciente foi atendida por três médicos generalistas, um oncologista e dois dermatologistas, sendo o primeiro atendimento um mês após o início da sintomatologia, porém sem conclusão diagnóstica até ser encaminhada ao serviço de dermatologia em 16/11/2017 e chegar ao diagnóstico final em 18/12/2017, depois de seis anos e sete meses de evolução. $\bigcirc$ exame dermatológico constatou duas placas eritemato-hipercrômicas infiltradas no abdômen, uma placa eritemato-hipercrômica descamativa com infiltração no MIE e um linfonodo palpável na região inguinal esquerda sob pele eritematosa (Figuras 1 e 2). Desde o início do quadro, foram realizados quatro exames histopatológicos e um imuno-histoquímico da lesão, evidenciando, por fim, infiltrado linfoide dérmico atípico com epidermotropismo, imunofenótipo T CD4 predominante, e com perda de expressão de CD7, sugerindo o diagnóstico de MF. Foram realizados tomografias computadorizadas de tórax e abdômen, que não apresentaram alterações significativas, e acompanhamento concomitante com a hematologia. Além do linfonodo clinicamente palpável, foi verificada linfonodomegalia na região inguinal direita, após ultrassonografia de partes moles, com múltiplos linfonodos acometidos, tendo - maior deles 3,2 x $1 \mathrm{~cm}$, além de espessamento de pele e densificação de gordura. Após avaliação, o estadiamento foi estabelecido como T2NxMOBO. Com isso, o tratamento proposto foi de metotrexato $15 \mathrm{mg}$ e ácido fólico $5 \mathrm{mg}$, em dose única semanal, e fototerapia com PUVA, iniciado em 22/02/2018. A paciente apresentou melhora significativa das lesões após o tratamento instituído, com regressão da infiltração, do eritema, da descamação e do gânglio, porém, com extensa área de atrofia (Figura 3) e infecções secundárias, apresentando dois episódios de erisipela e um de impetigo no membro afetado, com intervalos de quatro a seis meses entre eles. Os quadros de erisipela foram tratados com cefalexina $500 \mathrm{mg}$, via oral, de 6 em 6 h, por 14 dias, e o de impetigo, com ácido fusídico tópico, duas vezes ao dia, com melhora completa após a administração da antibioticoterapia.

\section{DISCUSSÃO}

A incidência anual de LCCT é extremamente rara, sendo de 0,77/100.000 indivíduos, e a MF, o tipo mais comum desse linfoma, possui incidência aproximada de 0,41/100.000 indivíduos².

A MF pode apresentar semelhança considerável com diversas dermatoses inflamatórias benignas, como eczema, psoríase e pitiríase liquenoide crônica, e suas características histológicas clássicas podem estar ausentes mesmo em múltiplas biópsias de pele, principalmente em seus estágios iniciais, como foi observado neste caso. Por conseguinte, o diagnóstico tardio pode resultar na evolução e agravamento do quadro do paciente, incluindo a piora em sua qualidade de vida, a necessidade de tratamentos mais agressivos, a ocorrência de sequelas e uma possível iatrogenia decorrente de exames e tratamentos anteriores ${ }^{3,45,8}$. Tal questão foi observada no presente relato, pois, em seis anos, a paciente consultou-se com seis médicos de diferentes especialidades até ser encaminhada ao serviço de dermatologia da UEPA e receber o diagnóstico definitivo. Essa demora resultou no agravamento do quadro e maior dificuldade terapêutica, além de grande despendimento de tempo e dinheiro. $\bigcirc$ atraso diagnóstico observado foi ainda 
maior do que o encontrado em estudos realizados por Skov e Gniadecki ${ }^{9}$ e Scarisbrick et al. ${ }^{10}$, em que o tempo médio do diagnóstico foi de dois anos e três meses e de três anos, respectivamente. Esses pontos

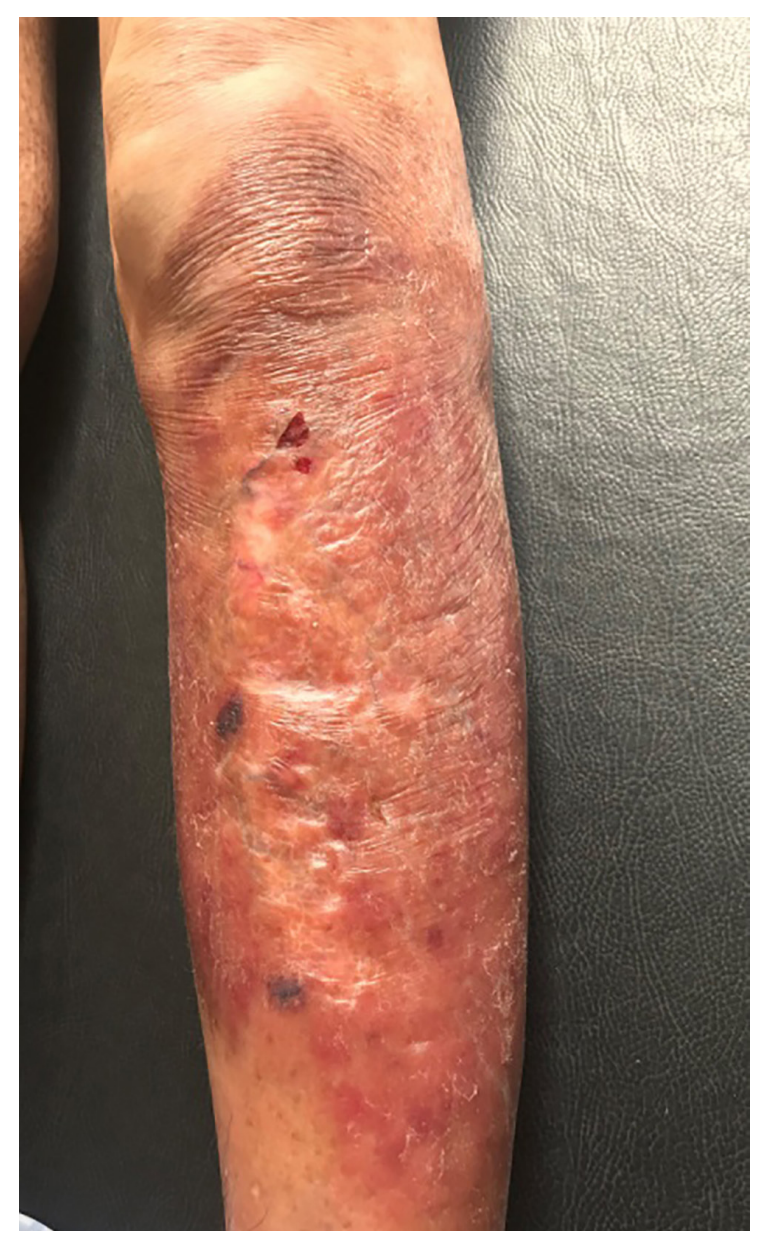

Foto: Brena Andrade de Lima Lobato.

Figura 1 - Placa eritemato-hipercrômica infiltrada com descamação e áreas exulceradas em membro inferior esquerdo reforçam o entendimento da MF como um problema de saúde pública, e a importância do profissional médico treinado para rastrear os casos antes de gerar maiores complicações.

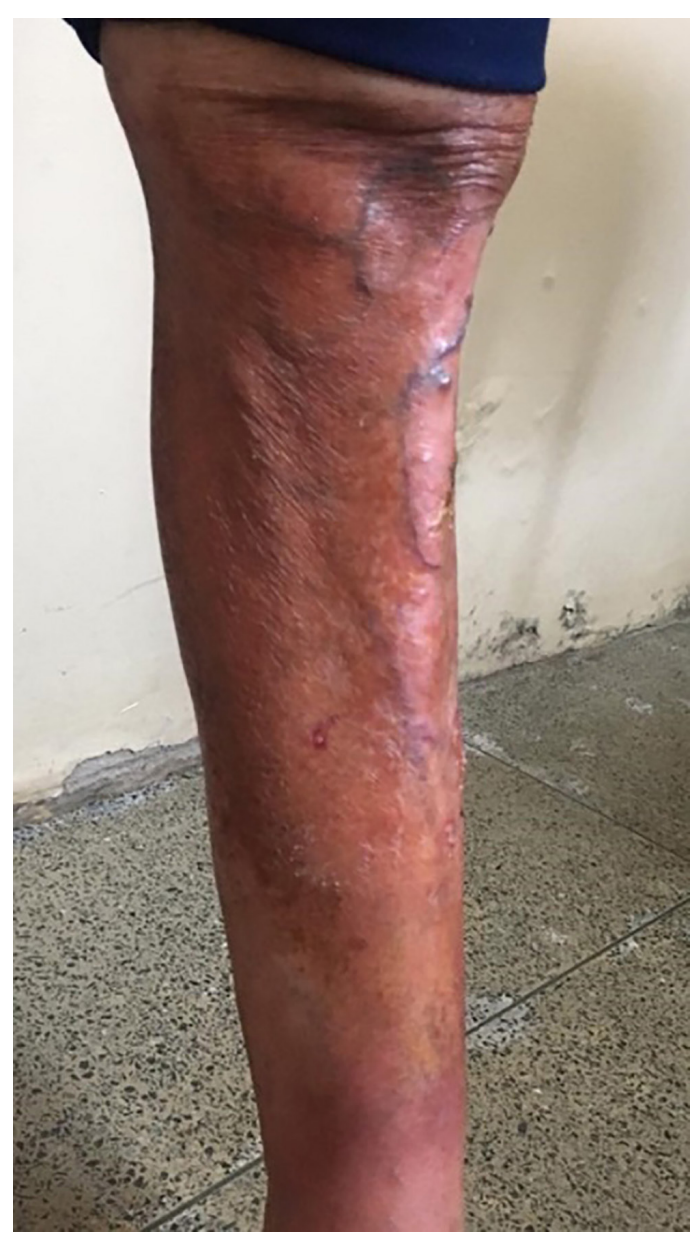

Foto: Brena Andrade de Lima Lobato.

Figura 3 - Mácula hipercrômica e área de atrofia em pele xerótica do membro inferior esquerdo, apresentando regressão da infiltração e do edema

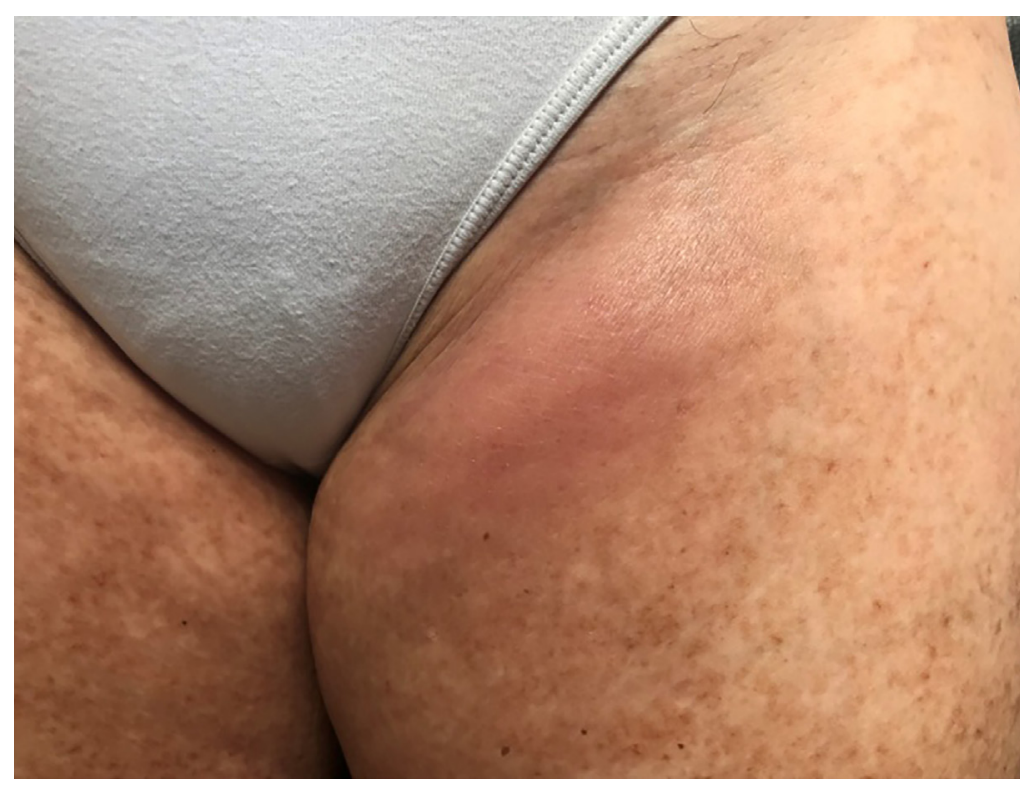

Foto: Brena Andrade de Lima Lobato.

Figura 2 - Nódulo endurecido e palpável sob pele eritematosa na região inguinal esquerda 
O estadiamento foi definido como T2NxMOBO pelo método tumor-node-metastasis (TNM) staging system, adaptado aos parâmetros específicos dos LCCT ${ }^{11}$. Assim, foi instituído o tratamento com metotrexato $15 \mathrm{mg}$ e ácido fólico 5 mg, em dose única por semana, associados à fototerapia com PUVA, não tendo sido observados efeitos adversos. $\bigcirc$ tratamento com fototerapia PUVA possui amplo benefício na remissão da MF, penetrando de forma profunda na derme e podendo ser utilizado em conjunto com agentes sistêmicos em casos mais graves ${ }^{7,12,13}$. $\bigcirc$ metotrexato, um análogo do ácido fólico que faz parte do grupo de drogas antimetabólicas, apresenta benefícios comprovados mesmo em baixas doses; porém, estudos comprovando sua utilização em ampla escala ainda são escassos ${ }^{14,15}$. Ademais, a associação do metorexato com a fototerapia apresentou resultados promissores no presente caso, reforçando a possibilidade de sua utilização como escolha terapêutica e objeto de estudos futuros.

\section{CONCLUSÃO}

A MF é uma doença linfoproliferativa cutânea de difícil diagnóstico, principalmente em estágios iniciais. $\bigcirc$ presente relato ressalta os impactos do atraso no diagnóstico e da dificuldade de profissionais médicos, de diferentes especialidades, em estabelecer esse diagnóstico, fatores que impactam na progressão do quadro, pioram o prognóstico, e podem gerar sequelas após o tratamento. Adicionalmente, mostra o dispêndio de tempo e recursos financeiros do paciente, o que afeta sua qualidade de vida. Diante do exposto, a MF é um grande desafio para a saúde pública, principalmente em relação à formação e capacitação de profissionais médicos.

\section{ASPECTOS ÉTICOS}

- presente relato de caso foi realizado durante a execução do projeto "Fototerapia em doenças linfoproliferativas e inflamatórias associadas ao HTLV", o qual foi aprovado pelo Comitê de Ética em Pesquisa da Universidade do Estado do Pará, com parecer $\mathrm{n}^{\circ}$ 1.582.819, em 9 de junho de 2016, e está em consonância com os preceitos da Declaração de Helsinque e do Código de Nuremberg, respeitando as normas de pesquisa envolvendo seres humanos, conforme a Resolução $n^{\circ} 466 / 12$ do Conselho Nacional de Saúde.

\section{CONFLITOS DE INTERESSE}

Os autores declaram não haver conflitos de interesse.

\section{CONTRIBUIÇÃO DOS AUTORES}

Todos os autores contribuíram integralmente com a elaboração do presente relato de caso, realizando: coleta de dados, acompanhamento e manejo clínico do caso, idealização do relato, revisão crítica da literatura e dos dados obtidos, redação do manuscrito e aprovação da versão final.

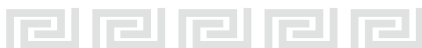

\section{REFERÊNCIAS}

1 Willemze R, Jaffe ES, Burg G, Cerroni L, Berti E, Swerdlow SH, et al. WHO-EORTC classification for cutaneous lymphomas. Blood. 2005 May; 105(10):3768-85.

2 Bradford PT, Devesa SS, Anderson WF, Toro JR. Cutaneous lymphoma incidence patterns in the United States: a population-based study of 3884 cases. Blood. 2009 May; 1 13(21):5064-73.

3 Jawed SI, Myskowski PL, Horwitz S, Moskowitz A, Querfeld C. Primary cutaneous T-cell lymphoma (mycosis fungoides and Sézary syndrome): part I. Diagnosis: clinical and histopathologic features and new molecular and biologic markers. J Am Acad Dermatol. 2014 Feb;70(2):205.e1-16.

4 Foss FM, Girardi M. Mycosis fungoides and Sezary syndrome. Hematol Oncol Clin North Am. 2017 Apr;31 (2):297-315.

5 Larocca C, Kupper T. Mycosis fungoides and Sézary syndrome: an update. Hematol Oncol Clin North Am. 2019 Feb;33(1):103-120.

6 Whittaker S, Hoppe R, Prince HM. How I treat mycosis fungoides and Sézary syndrome. Blood. 2016 Jun; 127(25):3142-53.
7 Lovgren ML, Scarisbrick JJ. Update on skin directed therapies in mycosis fungoides. Chin Clin Oncol. 2019 Feb;8(1):7.

8 Jawed SI, Myskowski PL, Horwitz S, Moskowitz A, Querfeld C. Primary cutaneous T-cell lymphoma (mycosis fungoides and Sézary syndrome): part II. Prognosis, management, and future directions. J Am Acad Dermatol. 2014 Feb;70(2):223.e1-17.

9 Skov AG, Gniadecki R. Delay in the histopathologic diagnosis of mycosis fungoides. Acta Derm Venereol. 2015 Apr;95(4):472-5.

10 Scarisbrick JJ, Quaglino P, Prince HM, Papadavid E, Hodak E, Bagot $M$, et al. The PROCLIPI international registry of early-stage mycosis fungoides identifies substantial diagnostic delay in most patients. $\mathrm{Br} J$ Dermatol. 2019 Aug; 181 (2):350-7.

11 Olsen E, Vonderheid E, Pimpinelli N, Willemze R, Kim Y, Knobler $R$, et al. Revisions to the staging and classification of mycosis fungoides and Sezary syndrome: a proposal of the International Society for Cutaneous Lymphomas (ISCL) and the cutaneous lymphoma task force of the European Organization of Research and Treatment of Cancer (EORTC). Blood. 2007 Sep; 1 10(6):1713-22. 
12 Trautinger F. Phototherapy of cutaneous T-cell lymphomas. Photochem Photobiol Sci. 2018 Dec;17(12):1904-12.

13 Olsen EA, Hodak E, Anderson T, Carter JB, Henderson M, Cooper K, et al. Guidelines for phototherapy of mycosis fungoides and Sézary syndrome: a consensus statement of the United States Cutaneous Lymphoma Consortium. J Am Acad Dermatol. 2016 Jan;74(1): 27-58.
14 Olek-Hrab K, Mai J, Chmielowska E, Jankowska-Konsur A, Olszewska B, Kręcisz B, et al. Methotrexate in the treatment of mycosis fungoides - a multicenter observational study in 79 patients. Eur Rev Med Pharmacol Sci. 2018 Jun;22( 11 1):3586-94.

15 Photiou L, van der Weyden C, McCormack C, Prince HM. Systemic treatment options for advanced-stage mycosis fungoides and Sézary syndrome. Curr Oncol Rep. 2018 Mar;20(4):32.

Recebido em / Received: 22/10/2020 Aceito em / Accepted: 1/6/2021 